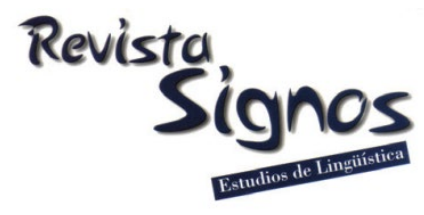

\title{
Estudio de variación en el uso de atenuación II: Microanálisis de secuencias discursivas, actos de habla y recursos atenuantes*
}

\section{$V$ ariationist study of mitigation II: Microanalysis of discourse sequences, speech acts and mitigating resources}

\author{
Marta Albelda Marco \\ UNIVERSITAT DE VALÈNCIA \\ ESPAÑA \\ marta.albelda@uv.es
}

\author{
Ana María Cestero Mancera \\ UNIVERSIDAD DE ALCALÁ \\ ESPAÑA \\ anam.cestero@uah.es
}

Recibido: 17-I-2020 / Aceptado: 21-VII-2020

DOI: $10.4067 /$ S0718-09342020000300962

\section{Resumen}

El estudio de la atenuación pragmática ha sido priorizado en los últimos años, especialmente en las disciplinas que trabajan con el habla, como el análisis del discurso y de la conversación. Sin embargo, la variabilidad sociopragmática y geolectal en usos y recursos de atenuación, al menos para el caso del español, apenas se ha abordado. A ello se dedica el presente trabajo, que se complementa con la investigación presentada en el artículo de Cestero y Albelda (2020); así se pretende dar cuenta del estado actual de la investigación variacionista sobre la estrategia atenuadora, especialmente desarrollada en el marco del Proyecto para el estudio sociolingüístico del español de España y América (PRESEEA). En el presente artículo se atiende, primero, al microanálisis de determinadas secuencias y actos de habla y de algunos recursos específicos de atenuación, y segundo a la comparación de los resultados de atenuación en PRESEEA con los datos obtenidos de otros tipos de corpus discursivos. Todo ello permite apuntar datos de variación no solo sociopragmática y geolectal sino también diafásica.

Palabras Clave: Atenuación, variación diafásica, variación geolectal, PRESEEA, español hablado. 


\begin{abstract}
The study of pragmatic mitigation has been prioritized in the last decades, particularly in speech-related disciplines such as discourse analysis and conversation analysis. Nevertheless, the sociopragmatic and geolectal variability of mitigation in Spanish has barely been studied. This is the main objective of this paper (which complements the research presented in Cestero \& Albelda, 2020), which aims to examine the state of the art on sociolinguistic variation in mitigation strategies, especially within the framework of the Project for the Sociolinguistic Study of Spanish in Spain and America (PRESEEA). The present article addresses two further aspects: a study in greater depth of some discourse sequences and speech acts and some specific mitigation resources, and a diaphasic comparison between the results obtained from the PRESEEA corpus and those from other corpora. The findings of the present report suggest that variations in mitigation are not only dialectal and sociolectal but also diaphasic.
\end{abstract}

Key Words: Mitigation, diaphasic variation, dialectal variation, PRESEEA, spoken Spanish.

\title{
INTRODUCCIÓN
}

En esta última década se ha desarrollado el estudio sociolingüístico y dialectal de la atenuación, especialmente en el marco del proyecto internacional PRESEEA (véase al respecto también el artículo de Cestero \& Albelda (2020). El establecimiento de una ficha detallada para su análisis cualitativo y codificación (Albelda \& Cestero, 2011, Cestero \& Albelda, 2012; Albelda, Briz, Cestero, Kotwica \& Villalba, 2014), así como la elaboración de una Guía de estudios de la atenuación (Cestero \& Rodríguez, 2014), en la que se exponen los procedimientos analíticos, ha permitido su estudio en profundidad y, a partir de los resultados, la descripción progresiva de patrones sociopragmáticos. La propuesta de trabajo coordinado de PRESEEA (2003, 2008), por su parte, ha propiciado la unificación metodológica y, con ella, la comparabilidad de datos, lo que nos está llevando al establecimiento de patrones dialectales y a la identificación de variación diafásica.

En el artículo que precede y complementa este trabajo (parte I, en este volumen), se ofrece una caracterización actualizada de la atenuación y se detalla la metodología de análisis; además, se esbozan patrones sociopragmáticos y dialectales de la atenuación en español, gracias a la revisión, el análisis y la comparación de los resultados obtenidos ya en los análisis completos de la estrategia en corpus del PRESEEA. Hasta ahora, se han podido delinear patrones, por contarse con estudios completos sobre la atenuación, en España, de Madrid, Valencia, Las Palmas de Gran Canaria y, parcialmente, Granada, y en Hispanoamérica, de Santiago de Chile y Puebla de Zaragoza, y parcialmente, de Monterrey.

El presente artículo se dedica, en un primer momento, a revisar, analizar, unificar y comparar los resultados de los trabajos que se centran en microanálisis particulares estudios en profundidad- de algunos tipos de secuencias textuales y actos de habla 
(apartado 1), así como de determinados recursos de atenuación (apartado 2), esto es, a lo que se ha considerado modalidad B de análisis de PRESEEA_ATENUACIÓN. Cabe recordar que las secuencias textuales recogidas en la ficha de atenuación PRESEEA, como se ha visto en la parte I (sección 2.2.), son argumentación, exposición, narración y descripción. Los recursos de atenuación también se expusieron en el artículo anterior (sección 2.2.), agrupados en siete categorías más generales que se gradúan en una escala de más a menos involucramiento del hablante y en la que su imagen se expone cada vez menos: (1) corrección o reparación de lo dicho o hecho; (2) acotación de lo dicho o hecho; (3) reducción de lo dicho; (4) minimización y/o difuminación de la cantidad o cualidad de lo dicho; (5) justificación; (6) implicación del tú en lo dicho o hecho, y (7) impersonalización o desfocalización. En esta ocasión, trabajamos con datos de más comunidades, concretamente, de Monterrey, Barranquilla, Medellín, La Habana, Caracas, Mérida y Santiago de Chile, en Hispanoamérica, y de Madrid, Valencia, Sevilla y Granada, en España.

Por último, se atiende y se da cuenta aquí de aquellos estudios que comparan los resultados del estudio de la atenuación en entrevistas en registro medio de lengua, propias de los corpus PRESEEA, con los datos obtenidos en otros tipos de corpus discursivos (apartado 3), con lo que iniciamos la identificación de variación diafásica. Los trabajos aquí revisados son solo aquellos en los que se trabaja con corpus recogidos con control sociolingüístico similar al de PRESEEA, pues se considera necesario para establecer comparaciones, y son, en su mayoría, corpus de conversaciones en registro informal (Briz \& Grupo Val.Es.Co., 2002; Albelda \& Estellés, en línea).

El establecimiento del estado de la cuestión que hemos llevado a cabo nos ha permitido apuntar datos de variación sociopragmática y geolectal y dar cuenta de la existencia de variación diafásica y de posibles procesos de cambio, además de continuar perfilando patrones sociolectales y dialectales.

\section{La atenuación en secuencias discursivas y actos de habla}

En el marco de PRESEEA_ATENUACIÓN, se ha atendido, con un acercamiento microanalítico, al uso, a los recursos y al funcionamiento sociopragmático de la atenuación en algunas clases de secuencias discursivas o tipos textuales concretos, de los que se obtienen muestras por la configuración misma de las encuestas semidirigidas a través de módulos temáticos determinados, así como de distintos tipos de actos de habla o fenómenos pragmáticos concretos. En estos casos, no se lleva a cabo un estudio completo de la atenuación en todo el corpus sociolectal y dialectal, sino que se trabaja, en profundidad, sobre los patrones sociopragmáticos obtenidos de extractos conformados por las secuencias o los actos de habla objeto de investigación.

Esta modalidad de investigación de la atenuación se ha desarrollado, de manera especial, en el seno del equipo PRESEEA-Monterrey (México), que ha ofrecido ya 
datos de gran interés sobre patrones sociopragmáticos de la atenuación en secuencias argumentativas (Rodríguez Alfano, 2016, 2018), en ironía (Flores Treviño, 2013, 2016a) y en actos asertivos (Flores \& Ramírez, 2015). En PRESEEA-Barranquilla (Colombia), se ha investigado la atenuación también en argumentación y se le ha añadido la secuencia narrativa, lo que nos ha proporcionado los primeros hallazgos sobre la atenuación en la ciudad colombiana (Torres \& Rodríguez, 2017). El funcionamiento de la atenuación en este último tipo de secuencia se conoce bien en PRESEEA-Santiago de Chile gracias al trabajo de González Riffo (2017). Además, disponemos de un primer acercamiento al uso y funcionamiento de la atenuación en el habla de La Habana gracias al estudio de los actos de habla disentivos realizado por Blanco (2014). Ofrecemos, a continuación, los datos de los que hemos podido disponer hasta ahora y que completan el conocimiento sobre la atenuación y su funcionamiento en interacción comunicativa en registro medio de lengua.

En primer lugar, veamos la atenuación en secuencias discursivas irónicas. La ironía, como es bien sabido, se considera un fenómeno pragmático de gran rendimiento funcional en la interacción comunicativa. Se relaciona muy directamente con la atenuación, pues los actos irónicos, en gran medida, se realizan a través de mecanismos mitigadores, que estratégicamente protegen la imagen del propio hablante o previenen una amenaza a la imagen de los interlocutores. Ello se puede ver claramente en los resultados de Flores Treviño (2013, 2016a) en el Habla de Monterrey (México), los cuales, tras analizar 12 entrevistas, 6 de mujeres y 6 de hombres, mayores y con nivel educativo alto, muestran que la mayoría de las ironías del corpus, el 77,1\%, se realizan con atenuación (Flores Treviño, 2016a). Y, en consonancia con los patrones sociopragmáticos mexicanos presentados en el artículo de la parte I de esta investigación, los hombres atenúan al ironizar mucho más que las mujeres $(72,4 \%$ frente a 27,5\%). Parece que, en estos casos, la estrategia más asidua es la de desfocalización mediante impersonalización, es decir, la de mínima exposición de la imagen propia, lo que se relaciona con la función de autoprotección que, según apunta Flores Treviño (2016a), en la muestra analizada es la mayoritaria, de nuevo en consonancia con los patrones sociopragmáticos de la atenuación en la interacción mexicana (artículo de la parte I).

Un tipo de actos que implica, de manera directa, la exposición de la imagen propia, y que, por tanto, se relaciona con la atenuación, es el asertivo, predominante en las entrevistas semidirigidas de PRESEEA-Monterrey por su configuración estructural y temática. Flores y Ramírez (2015) abordaron su estudio bajo la hipótesis de ser actos a través de los que se exponen creencias, juicios de valor y puntos de vista propios y en los que, por tanto, se presenta y proyecta una imagen y, en ocasiones, puede afectar a la de 'otros'. Los resultados del análisis de los actos asertivos de 20 minutos de 12 entrevistas, 6 de hombres y 6 de mujeres, adultos con estudios superiores, han permitido constatar la productividad discursiva de la atenuación en esta ciudad y 
considerar que dicha estrategia contribuye a caracterizar la imagen de afiliación de los hablantes (Flores \& Ramírez, 2015). Además, los datos anteriores arrojan más luz sobre patrones sociopragmáticos en la urbe mexicana, en parte, ahora, diferentes a los documentados por Palacios (en prensa) en Puebla, lo que podría indicar cierta identidad cultural. En Monterrey, en actos asertivos, las mujeres adultas atenúan más que los hombres (Flores \& Ramírez, 2015). Ambos atenúan preferiblemente justificando e implicando al interlocutor (Flores \& Ramírez, 2015), estrategias que ocupan los lugares intermedios del continuum de exposición de la imagen, pero hacia el polo del distanciamiento y la desfocalización. Ahora bien, el estilo interactivo de hombres y mujeres al respecto marca diferencias sociopragmáticas claras, pues los hombres, como en otras comunidades, prefieren atenuar desfocalizando (así lo hacen en el 31\% de las ocasiones, frente al 10\% en el que usan las mujeres esta estrategia), polo de mínima exposición de imagen, mientras que las mujeres muestran una predilección por la justificación y explicación (constituye el 28\% de sus empleos de atenuación, frente al $17 \%$ en que lo usan los hombres), punto intermedio en exposición (Flores \& Ramírez, 2015).

En el habla de Santiago de Chile (corpus PRESEEA), Guerrero, Gajardo, González y Reyes (en prensa), tomando como base la investigación de Flores y Ramírez (2015), han iniciado el estudio de los recursos para justificar en actos asertivos. Por el momento, los autores ofrecen una propuesta teórico-metodológica detallada y los primeros resultados sobre el funcionamiento pragmático del acto de justificación en la capital chilena.

Por último, se ha estudiado, en profundidad, el funcionamiento sociopragmático de la atenuación en justificaciones argumentativas en el habla de Monterrey (Rodríguez Alfano, 2016, 2018), lo que ha permitido obtener datos sobre el fenómeno que nos ocupa, que complementan los previos y aumentan nuestro conocimiento, a la par que suponen un avance considerable en su investigación para el establecimiento de patrones sociolingüísticos y geolectales. Rodríguez Alfano analiza 30 minutos de las 108 entrevistas que componen el corpus PRESEEA-Monterrey, 6 hombres y 6 mujeres por grupo etario y nivel de instrucción, y documenta en la muestra 698 justificaciones atenuadas, que suponen el $82 \%$ de las secuencias objeto de estudio identificadas, lo que permite concluir que se trata de una estrategia característica de la justificación argumentativa, al menos en la urbe mexicana, consideración que se refuerza si se tiene en cuenta el número total de recursos de atenuación hallados: 5891 (Rodríguez Alfano, 2018). Una vez más, ahora en secuencias de justificación argumentativa, los resultados indican que los hombres atenúan más que las mujeres (ellos han producido el 58,4\% de los recursos identificados, frente al 41,6\% empleados por ellas) y que los jóvenes lo hacen más que los mayores y que los adultos $(40,60 \%$, $30,40 \%$ y 29\%, respectivamente), como ocurría en Puebla (parte I). Sin embargo, el patrón no se repite en relación con el nivel educacional, pues, en Monterrey 
(Rodríguez Alfano, 2018), la frecuencia de utilización de atenuación disminuye a medida que aumenta el nivel de instrucción de los sujetos, siendo los más instruidos los que menos acuden a la estrategia (39\%, 35\% y 26\%, respectivamente). En relación con los recursos empleados mayoritariamente, la investigadora resalta la preferencia de los hombres jóvenes con nivel de instrucción media por procedimientos que inciden en la franqueza de lo dicho, objetivándolo, lo que lleva al polo de menor exposición de la imagen del continuum establecido, aunque también alude al uso frecuente de partículas de reformulación, que coloca a los sujetos en el lugar de la exposición máxima (Rodríguez Alfano, 2018), con un balanceo que podría ser característico de la ciudad nororiental mexicana.

Los únicos datos del funcionamiento de la atenuación en una ciudad colombiana a los que hemos podido acceder son los de Torres y Rodríguez (2017) como resultado del estudio sobre secuencias argumentativas y narrativas del corpus PRESEEABarranquilla. Las investigadoras analizan los 10 primeros minutos de las 72 entrevistas del corpus barranquillero, 4 de hombres y 4 de mujeres, de cada uno de los tres grupos etarios y de los tres niveles educativos establecidos, y obtienen resultados de gran interés, a partir de los cuales puede vislumbrarse una primera aproximación a los patrones sociopragmáticos del fenómeno que nos ocupa, que individualiza la comunidad de habla y le confiere identidad. Como ya comprobara Rodríguez Alfano en su estudio de las justificaciones argumentativas en Monterrey, es muy frecuente atenuar en secuencias argumentativas, pues exponen imágenes, especialmente la del 'yo'; así se confirma también en el habla de Barranquilla, en donde la atenuación en argumentación es significativamente mucho más frecuente que en narración (Torres \& Rodríguez, 2017). Como se ha documentado en las otras urbes americanas estudiadas de manera completa y en la capital española (parte I), en la ciudad colombiana emplean más recursos de atenuación los hombres que las mujeres, si bien la diferencia es mínima, de un solo punto, y la utilización de recursos disminuye a medida que aumenta la edad, como en Santiago de Chile, en Puebla, en Madrid y en Las Palmas, y se incrementa cuando sube el nivel de instrucción de los sujetos (Torres \& Rodríguez, 2017), también como en Madrid y Las Palmas, y Puebla, pero no como en Santiago de Chile o en Valencia. Las estrategias, de nuevo, se mueven en un balanceo entre los dos polos del continuum de exposición de la imagen, pues el recurso más usado es la impersonalización, seguido de los retardadores, la modificación externa e interna y la expresión de la aserción en forma de duda, lo que lleva a pensar en la desfocalización como estrategia atenuadora más característica de los barranquilleros, especialmente en el caso de los hombres con bajo o alto nivel de instrucción (Torres \& Rodríguez, 2017).

La narración, como se acaba de mencionar, presenta mucha menos atenuación que otras secuencias discursivas como la argumentación; no obstante, también en ella es característico el fenómeno que tratamos, tal y como muestra el trabajo de González 
Riffo (2017) sobre estrategias de atenuación en narraciones de hablantes de Santiago de Chile. Contamos ya con los resultados del estudio general de la atenuación en PRESEEA-Santiago de Chile (Guerrero, en prensa; parte I). Los datos ofrecidos por González Riffo (2017) sobre lo que acontece en las narraciones permite complementarlos y confirmarlos, a la vez que ofrecen una información de especial interés, pues, como se detalla en el apartado 3 , se comparan los resultados del análisis de la atenuación en narración de dos corpus diferentes, el de entrevistas de PRESEEA y el denominado FONDECYT, de narraciones co-construidas. En el caso de la muestra de PRESEEA-Santiago de Chile, González Riffo (2017) analiza 36 narraciones de hombres y mujeres, de entre 35 y 54 años, de niveles de instrucción bajo, medio o alto. Las estrategias atenuadoras más asiduas son intermedias en el continuum de exposición de la imagen establecido (relacionadas con el eje que González Riffo (2017) llama 'estima', relacionado con recursos para minimizar, suavizar, apelar al interlocutor o justificar), a pesar de que lo usual es pensar que las estrategias atenuadoras en narraciones experienciales tienden a desenfocar, incidiendo en la veracidad y la certeza, hecho que podría relacionarse con los empleos más usuales de la atenuación en Santiago. Sin embargo, los resultados obtenidos con relación a la incidencia del nivel de instrucción y el sexo son distintos a los que ofrece Guerrero (en prensa), pues, en narraciones, las mujeres atenúan más que los hombres y los sujetos más instruidos atenúan más que el resto (González Riffo, 2017). Esto lleva a pensar que, en el tipo de secuencia investigada, los patrones se invierten, seguramente porque la función de la atenuación también es algo diferente, ya que estamos ante narraciones experienciales.

Por otro lado, los únicos datos que se tienen sobre el uso y funcionamiento sociopragmático de la atenuación en una urbe cubana, al menos hasta donde llega nuestro conocimiento, son los obtenidos por Blanco (2014) para La Habana, en su estudio de secuencias y actos de habla disentivos, manifestación del desacuerdo o la disconformidad. Como recuerda Blanco (2014), se trata de un tipo de acto despreferido que requiere, en cumplimiento de los principios interaccionales de cortesía y cooperación, señales que preparen al interlocutor para su recepción. En este sentido, la atenuación se presenta como una estrategia conveniente, que funciona distanciando al hablante de lo dicho o hecho, esto es, protegiendo su imagen, o enfocando la producción hacia el interlocutor, bien previniendo la amenaza o bien reparando la inadecuación. El análisis de Blanco sobre los actos disentivos de 36 encuestas del corpus PRESEEA-La Habana, 2 por hombres y 2 mujeres por cada grupo etario (jóvenes, adultos y mayores) y nivel de instrucción (alta, media y baja), le ha permitido comprobar que, en entrevista semidirigida en registro medio, las secuencias disentivas y los actos de habla disentivos en ellas son relativamente frecuentes, pues identificó 94 secuencias con 201 actos disentivos en total (Blanco, 2014: 60). Aunque se emplea más la intensificación, que resalta 'al otro', que la atenuación, que protege al 'yo', en las secuencias y actos, la atenuación se usa en un 
porcentaje no bajo, en un $38 \%$ de los actos, y para ello se emplean, sobre todo, movimientos para reducir al mínimo la disconformidad o que reflejan conformidad parcial como introducción del desacuerdo (Blanco, 2014), que sitúa la preferencia estratégica de los habaneros en puntos centrales y de mayor exposición de la imagen propia del continum y, en mucha menor medida, impersonalización, expresión de incertidumbre o fingimiento de ignorancia, lo que lleva al otro polo y lleva en ocasiones a la despersonalización. La diferencia en la producción de secuencias y actos disentivos de hombres y mujeres no es considerable, aunque los hombres producen más que las mujeres; sin embargo, sí podría ser significativo que sean los jóvenes los que, estratégicamente, atenúen más disentivos, pues así se ha documentado en más de la mitad de los casos, seguido, de lejos, por los mayores y por los adultos (Blanco, 2014). Finalmente, como ocurre en otras urbes estudiadas, son los sujetos de nivel de instrucción media los que más atenúan este tipo de actos, seguidos de los de nivel bajo y de los de alto (Blanco, 2014). Estos datos podrían llevar a pensar que se trata de una característica sociopragmática del estilo interaccional de hombres jóvenes y con instrucción media en La Habana. Esperamos tener pronto más datos que permitan constatar los patrones concretos en la comunidad caribeña.

\section{Variación sociopragmática y geolectal en el uso de recursos de atenuación}

En una tercera instancia, se ha atendido, desde un acercamiento microanalítico también, a recursos de atenuación concretos, cuyo uso parece resultar de interés en diferentes urbes hispanohablantes. Este tipo de investigaciones se incluyen en la modalidad B de PRESEEA_ATENUACIÓN y han proporcionado datos que informan sobre patrones sociopragmáticos, a la vez que abren el camino para realizar comparaciones interdialectales y establecer, a partir de ellas, tendencias geolectales, si se comprueba variabilidad diatópica. Como en los estudios microestructurales previos, quizás se pueda decir que el habla de Monterrey ha sido de las primeras en recibir atención en lo que respecta al estudio en profundidad de recursos de atenuación (Rivero, 2011, 2014; Silva, 2011; Reyes, 2014; González Salinas, 2015; Flores Treviño, 2016a, 2017), si bien es reseñable el estudio del diminutivo y de cuantificadores en el habla de Caracas también (Marcano, 2014; Malaver, 2018). España se ha incorporado a esta modalidad más recientemente y, en los últimos años, se ha atendido a lo que acontece con algunos recursos de atenuación en el habla de Granada (Montoro del Arco, 2011, 2012), de Madrid y Valencia (Estellés \& Cabedo, 2017; Uclés, 2018) o de Sevilla (León-Castro \& Repede, 2018; Repede \& León-Castro, 2019, en prensa; LeónCastro, 2019; Repede, 2019a, 2019b). Previamente se habían tratado también algunos mecanismos, como los diminutivos (Paredes, 2015; Uribe, 2017; Samper, 2019; Malaver \& Paredes, en prensa). el paralenguaje (Cestero, 2014), o los recursos fáticos de control de contacto (Cestero, 2019; Santana, 2019), si bien su estudio es más general y no se restringe únicamente a la función atenuadora. 
Rivero (2011, 2014), en un estudio sobre metáfora, metonimia y otras integraciones conceptuales y atenuación en el habla de Monterrey, para el que analiza narraciones de 12 entrevistas, 6 hombres y 6 mujeres, mayores e instruidos, concluye que la táctica de alusión no directa o a través de figuración a determinados temas comprometidos o tabú, esto es, eufemística, constituye una forma de suavizar acorde con la cortesía mexicana. La autora realiza un estudio de corte pragmático, no sociolingüístico, pero las ideas que aporta, especialmente las relacionadas con el uso muy frecuente de atenuación, cortés, en interacción, tanto con la función de autoprotección como de prevención, permiten constatar el patrón identificado en la urbe del noreste mexicano en estudios sociopragmáticos generales.

El trabajo de Reyes (2014) también muestra que los mexicanos de Monterrey usan frecuentemente la risa y el diminutivo como recursos de atenuación para autoproteger la imagen; de los dos, el primero es el más frecuente en la muestra analizada, entrevistas semidirigidas de 6 hombres y 6 mujeres instruidos, lo que podría explicar la tendencia al empleo de estrategia de corrección o reparación, en el extremo del continum de mayor exposición de imagen, que ello supone. La modificación morfológica atenuadora ya había sido atendida previamente por Silva (2011), también en un estudio sobre el corpus PRESEEA-Monterrey, casi exclusivamente pragmático, del que concluye que el extraordinariamente frecuente empleo del diminutivo con funciones diferentes de la referencial podría considerarse reflejo de un estilo 'emotivo', 'afectivo' y 'cortés' propio de los hablantes de la urbe mexicana (Silva, 2011). Por su parte, Flores Treviño (2016b) estudia la doble función pragmática de atenuación e intensificación que suele presentar el diminutivo de la forma 'bastante 'en este mismo corpus regiomontano. De acuerdo con la autora, 'bastantito' en esta variante del español imprime una intensificación semántica a la cualidad del elemento al que modifica y, al mismo tiempo, atenúa la fuerza ilocutiva del enunciado para la protección de la imagen de alguno de los interlocutores.

También otros signos paralingüísticos considerados atenuadores han recibido atención específica. Concretamente, nos referimos ahora a la prosodia, que es, seguramente, uno de los recursos de mayor rendimiento funcional, si bien aún no estudiado en profundidad desde la sociopragmática. Álvarez, Blondet y Rojas (2011) y Rojas, Blondet y Álvarez (2014) han comprobado cómo en el habla de Mérida (Venezuela), los hablantes parecen codificar la atenuación con tono alto, agudo, con un patrón $\mathrm{H}^{*}+\mathrm{L}$ como característico, y con una mayor duración de las sílabas atenuadas. Se trata aún de datos muy provisionales, pues el estudio es de caso, realizado sobre una única entrevista de PRESEEA-Mérida (de un hombre joven, con nivel de instrucción bajo), pero abre una vía de investigación que permite avanzar en el conocimiento de la forma y función, variables diatópicas, diastráticas y diafásicas, de la atenuación. 
Por su parte, Estellés y Cabedo (2017), en un estudio comparativo de la atenuación en entrevistas semiformales y conversaciones coloquiales, estudian los rasgos fónicos que expresan valores atenuantes, bien de manera autónoma, por sí solos (atenuación exclusivamente fónica) o en combinación con formas lingüísticas verbales (atenuación léxico-fónica). De acuerdo con los autores, los mecanismos fónicos más recurrentemente atenuantes son la reducción del tono de la voz y de la intensidad y, en segunda instancia, los alargamientos vocálicos y algunas modulaciones prosódicas singulares.

La risa, junto con otros signos paralingüísticos empleados como recursos de atenuación (bajada de tono y de volumen, vacilación, alargamiento de sonidos, elementos cuasi-léxicos), fue estudiada, en profundidad, por Cestero (2019) en una muestra de 18 entrevistas de PRESEEA-Madrid, 9 de hombres y 9 de mujeres, una por cada grupo etario y educativo establecidos, ya que se trata del recurso de atenuación más utilizado en la capital española (Cestero, 2014): aparece en más de la mitad de los actos atenuados, asiduamente en combinación con otros recursos lingüísticos. Los patrones sociopragmáticos de uso de signos paralingüísticos atenuadores en la urbe española confirman, por un lado, los apuntados como generales previamente, pero, por otro, dan cuenta de algunas especificidades que particularizan el empleo de los recursos que colocan a los hablantes en el polo de máxima exposición del continuum de atenuación. Así, si bien los hombres utilizan más que las mujeres este recurso en el corpus, como es lo normal en el patrón madrileño, ellas lo usan en mayor proporción y, también proporcionalmente, son más frecuentes en el habla de los mayores que de los jóvenes y adultos, y mayor en el nivel educativo medio y bajo que en el alto (Cestero, 2014).

El diminutivo, por su parte, ha sido uno de los recursos de atenuación más estudiados de todos los tiempos, si bien, en la revisión de los estudios sociopragmáticos y dialectales aquí tratados, su investigación se ha abordado como variable sociolingüística y dialectal, y no tanto por su carácter atenuador. En relación con la estrategia atenuadora, incluida entre los valores afectivos de la modificación morfológica, junto a la intensificación, los resultados obtenidos por Malaver y Paredes (en prensa; Paredes, 2015; Malaver, 2018) en los análisis sobre muestras de 72 entrevistas, 4 de hombres y 4 de mujeres por cada grupo etario y nivel de instrucción, de PRESEEA-Caracas, PRESEEA-Medellín y PRESEEA-Madrid aportan información relevante, pues, según documentan los investigadores, en Madrid se usa más el diminutivo con función evaluativa que en las urbes caribeñas, en contra de la creencia tradicional al respecto, y el efecto de los factores sociales en la función pragmática de los sufijos es escasa. Tales resultados acercan la urbe mexicana tratada previamente, salvando las distancias -en Monterrey el empleo del diminutivo es mucho más frecuente que en Madrid-, a la capital española y la distancian de otras comunidades hispanoamericanas, una vez más. 
En los últimos años, en la capital hispalense, se ha emprendido el estudio en profundidad del diminutivo, ahora sí centrándose, sobre todo, en su carácter atenuador (León-Castro, en prensa a, en prensa b). En los análisis realizados sobre 30 minutos de 72 entrevistas de PRESEEA-Sevilla, 4 de hombres y 4 de mujeres, de cada grupo de edad y nivel instruccional, León-Castro documenta un uso abundante de la modificación morfológica, especialmente en su forma -ito, que es empleada mayoritariamente como atenuador (59,2\% de los casos) y significativamente más por las mujeres (su habla presenta el 32,17\% de los diminutivos atenuadores, frente al $28,26 \%$ del habla de los hombres), los jóvenes (el 23\%, frente al $22 \%$ y al $15 \%$, por orden creciente) y las personas menos instruidas (28\%, frente a $17 \%$ y $16 \%$ ). Estos datos, al menos claramente los relativos a la incidencia del sexo y la edad, no se asemejan a lo que ocurre con la estrategia atenuadora en general en la capital española, y solo en lo referente al sexo -y en el empleo frecuente de este recurso- se podría relacionar con lo que acontece en la otra ciudad de variedad meridional estudiada hasta ahora, Las Palmas; por lo que, quizás, los resultados podrían reflejar un uso identitario o característico de la ciudad andaluza, que podrá comprobarse cuando se tengan resultados del estudio general de la atenuación en la urbe.

Estrechamente relacionados con los diminutivos, por aminorar cantidad o cualidad, pero, ahora mediante modificación externa, están los cuantificadores 'más o menos', 'medio' y 'un poco', estudiados en profundidad, sociopragmáticamente por Marcano (2014). La investigadora revisa su uso en el corpus PRESEEA-Caracas, de 72 entrevistas semidirigidas, lo que le permite documentar un uso frecuente en la capital venezolana, especialmente de 'un poco' (con o sin modificación morfológica interna). Se trata de un recurso utilizado más por hombres que por mujeres, por jóvenes que por adultos y mayores, y por sujetos con nivel de instrucción bajo que por los de instrucción media o alta. Estos datos podrían ser reflejo de un patrón general de estrategia en interacción comunicativa de los caraqueños, pendiente aún de comprobar, que muestra similitudes con lo que acontece en la capital española y en otras urbes americanas estudiadas, pero que podrían, a su vez, individualizar la comunidad caribeña.

Otro elemento de modificación externa estudiado sociolingüísticamente es 'como' y su variante 'como que'. Entre otros estudios, se puede mencionar el trabajo de Panussis y San Martín (2017) en el que, sobre un corpus sociolingǘstico de 72 entrevistas del habla de Santiago de Chile (ESECH), que sigue unos parámetros de estratificación muy cercanos a los de PRESEEA, se ha obtenido que el 68,7\% de los usos de 'como (que)' presenta valores atenuantes y aproximativos. Asimismo, esta función atenuante del 'como' es más frecuente en mujeres que en hombres, más abundante a medida que la edad se reduce, es decir, en la franja etaria más joven, y más común en niveles socioeconómicos más altos que en bajos. Un estudio similar sobre 'como (que)' es el de Mondaca (2017), ahora en un corpus de diversas ciudades 
de Chile, integrado por 24 entrevistas sociolingüísticas, cuyos informantes se encuentran en un tramo etario de entre 18 y 24 años. En su análisis se obtuvieron 1268 usos de 'como (que)' atenuante, distribuidos en un $62 \%$ en mujeres y en un $38 \%$ en hombres.

Y también en cierta medida relacionados con los diminutivos, por tratarse de operadores que, entre otras funciones, minimizan lo dicho o hecho, están los modificadores externos catalogados como marcadores de foco de exclusión. Su estudio, considerando la función atenuadora entre otras, fue abordado hace ya una década por Montoro del Arco $(2011,2012)$, quien sentó las bases para su investigación sociopragmática posterior y, además, ofreció datos importantes de variación geolectal. El investigador analiza las unidades monoverbales y pluriverbales de uso frecuente 'solo', 'solamente', 'nada más', 'nada más' que en los corpus PRESEEA-Granada y PRESEEA-Valencia y concluye que el discurso de los andaluces de Granada puede ser tildado de más expresivo que el de los valencianos, basándose en la mayor presencia en él de variantes fraseológicas y en el empleo mayoritario de estos operadores con valores pragmáticos, en mayor proporción en Granada que en Valencia el atenuador, lo que acerca Granada a lo que acontece en el uso general de la atenuación en otra comunidad meridional como Las Palmas, donde la modificación externa es el recurso más empleado como atenuador. En el caso de Granada, además, el nivel de instrucción de los hablantes se revela como factor de gran importancia en la elección y uso de las variantes en cuestión, lo que lleva a pensar en el menor prestigio que parece tener la unidad fraseológica (Montoro, 2011).

Teniendo en cuenta la propuesta de investigación de Montoro del Arco (2011, 2012), Flores Treviño (2017) estudia, en profundidad, los marcadores de foco de exclusión atenuadores en el habla de Monterrey y obtiene resultados de su funcionamiento sociopragmático de gran interés. Aunque el objetivo último del trabajo es comparar el rendimiento funcional de los marcadores en dos tipos de actividades interactivas distintas, la entrevista semidirigida y la conversación, que comentaremos en el siguiente apartado, ofrece datos relevantes hallados en el análisis de las 108 encuestas de PRESEEA-Monterrey, 6 de hombres y 6 de mujeres por cada grupo etario y nivel educativo, y que permiten confirmar los patrones característicos del habla mexicana en relación con la atenuación, pues Flores Treviño (2017) constata la alta frecuencia de uso de estos marcadores, especialmente con la función de autoprotección de imagen -si bien en un relativamente alto porcentaje para prevenir una amenaza a la imagen de otros- y en actos de habla que desfocalizan mediante impersonalización o corrigen y reparan ${ }^{1}$ (Flores Treviño, 2017). Hemos de esperar al estudio sociopragmático de estos elementos para establecer los patrones significativos de su uso geolectal. De la misma manera, hemos de esperar a tener datos sociopragmáticos para conocer posibles patrones en el uso de la unidad no sé como atenuadora. González Salinas (2015) ha iniciado su estudio en el habla de Monterrey 
hace unos años ya, si bien, de momento, solo disponemos de resultados que apuntan a un uso frecuente estable en entrevistas semidirigidas, de mujeres y hombres, jóvenes e instruidos, cuya función mayoritaria es, de nuevo, la autoprotección de la imagen.

De entre los recursos que se sitúan hacia el polo de menor exposición de la imagen del continum formulado en el marco teórico, en el artículo previo, en relación con los patrones sociopragmáticos y geolectales de la estrategia atenuadora, se han estudiado en profundidad, recientemente, los apéndices interrogativos de control de contacto y diversas formas de impersonalización, sobre todo, esta vez, en variedades de urbes españolas. Uclés (2018) ha atendido a los apéndices que implican al tú como estrategia atenuadora con objeto de comprobar variación geolectal; para ello, ha analizado dos entrevistas, una de un hombre instruido y otra de una mujer instruida, de los corpus PRESEEA-Madrid, PRESEEA-Valencia, PRESEEA-Ciudad de México y PRESEEAMonterrey, lo que le ha permitido constatar que se trata de marcadores utilizados más en México y en Madrid que en las otras dos urbes, y que su funcionamiento es muy similar en las comunidades estudiadas. A este respecto, cabe recordar que '¿no?' se ha revelado como el apéndice de uso más general en los dos lados del Atlántico, '¿eh?' como un recurso más propio de geolectos españoles, ‘¿sabes?' como marcador sociolingüístico madrileño y ‘ ¿verdad?’ como marcador característico mexicano. Cabe mencionar también aquí los trabajos de Cestero (2019) y de Santana (2019) sobre estos apéndices en el habla de Madrid y Sevilla, respectivamente, si bien, en estos casos, aún no se han estudiado en profundidad los recursos como atenuadores, sino que se atiende a la función como una dimensión de modalización entre otras, por lo que no contamos con datos que posibiliten el establecimiento de patrones sociopragmáticos y geolectales. En Madrid, según Cestero (2019), se emplean los apeladores al interlocutor como recursos intensificadores en una proporción alta (40,3\% de los casos), aunque es más frecuente que ni atenúen ni intensifiquen, datos que resultan acordes con el hecho de que los madrileños prefieran estrategias atenuadoras que exponen más la imagen propia, del otro polo del continuum. Por su parte, Santana (2019) está llevando a cabo el estudio de estos recursos en el habla de Sevilla; como en el caso de Madrid, no tenemos información completa que dé cuenta de su uso y funcionamiento sociopragmático todavía, pero la investigadora ha obtenido ya resultados en el habla de los sujetos menos y más instruidos de Sevilla, que resultan de interés y podrían adelantar patrones, pues comprueba claramente que los hombres sevillanos usan más que las mujeres el apéndice de uso frecuente '¿no?' como atenuante.

En el extremo de mínima exposición de la imagen del continuum están las impersonalizaciones y otros recursos de desfocalización, que se han documentado ya como los de uso más frecuente en Valencia, como de uso muy frecuente, aunque balanceado, en Puebla (México) y como de uso relativamente frecuente en Las Palmas y en Madrid. León-Castro y Repede han estudiado, en profundidad, el empleo de 'uno' 
atenuador en Sevilla y Granada, lo que nos ha proporcionado datos de gran interés sobre el impersonalizador (León-Castro \& Repede, 2018; León-Castro, 2019; Repede \& León-Castro, 2019). Las investigadoras han constatado, en los análisis realizados sobre los corpus PRESEEA-Sevilla y PRESEEA-Granada completos, que se trata de un recurso atenuador de gran productividad en las dos comunidades andaluzas, más usado por hombres que por mujeres, especialmente en Granada, donde corresponde a los hombres el 76\% de su uso, por los mayores que por los adultos y los jóvenes, y por los sujetos más instruidos (León-Castro, 2019), datos que se asemejan a los obtenidos en el uso de atenuación, en general, en Madrid, en relación al sexo, y en Madrid y Las Palmas con respecto a la edad. Por otra parte, lo que podría caracterizar, individualizando, el habla de las dos urbes es que los granadinos parecen usar 'uno' sobre todo para ocultarse, por tanto, con un patrón de menos desfocalización, mientras que los sevillanos hacen con él generalizaciones inclusivas, con un patrón de desfocalización intermedia (León-Castro, 2019). Repede y León-Castro (en prensa) han analizado también el empleo de 'se' y 'tú 'impersonalizadores en las 24 entrevistas semidirigidas del sociolecto alto de PRESEEA-Sevilla, y, aunque el estudio sociopragmático aún no está completo, los resultados muestran que se trata de unidades más o menos usadas en igual proporción por hombres y por mujeres y de predominio en el habla de los adultos; de ellas, los jóvenes instruidos prefieren 'tú' y los mayores, 'se'. Y no podemos olvidar, finalmente, el estudio en profundidad del discurso reproducido como atenuador realizado por Repede sobre el corpus PRESEEA-Sevilla (Repede, 2019a, 2019b), que le ha llevado a identificar 268 casos de discurso referido empleado como recurso atenuador, de desfocalización (sobre todo con el mecanismo de impersonalización y despersonalización, y menos de enajenación, por lo que no en el grado mayor de distanciamiento), en las 72 entrevistas semidirigidas de la capital andaluza. De nuevo, se trata de un recurso igualmente usado por hombres y por mujeres, y de predominio en el habla de los adultos (que prefieren la despersonalización a la impersonalización, más propia de los jóvenes, y a la enajenación, más característica de los mayores) y, en este caso, más asiduo en las interacciones de los sujetos con nivel de instrucción bajo (que muestran preferencia por la impersonalización), que medio (que prefieren la despersonalización) y que alto (que son lo usan en mayor proporción como enajenación) (Repede, 2019b).

Por último, se han estudiado los valores atenuantes de los evidenciales en diversos corpus PRESEEA de España (Estellés \& Albelda, 2017), aunque aún no se ha atendido expresamente a la variación sociolectal. Las autoras analizan las partículas 'por lo visto' y 'al parecer' en las entrevistas PRESEEA-Valencia y PRESEEAGranada y han contrastado los resultados con los de otros corpus de conversaciones y debates parlamentarios. El estudio muestra no solo la variación dialectal y genérica del uso de estos elementos, sino también la posibilidad de expresar otros valores más allá del atenuante en función de su contexto particular. Se le dedica más atención en el 
siguiente apartado, en el que se atiende a lo que acontece tanto en los diversos registros de lengua, mediante la comparación de resultados de muestras de PRESEEA y de otros corpus de habla.

\section{Estudio sociolingüístico de la atenuación en distintos tipos de actividades comunicativas: Entrevistas semidirigidas y conversaciones}

Como ya han apuntado trabajos previos (Estellés \& Albelda, 2017; Albelda \& Mihatsch, 2017), la atenuación es un fenómeno que ofrece también variación genérica (respecto al género discursivo) y diafásica (respecto al registro de habla). Se dedica a ello la última parte este trabajo sobre patrones sociolingüísticos y geolectales de la atenuación, dando cuenta de los hallazgos obtenidos al comparar lo que acontece en muestras de habla de corpus PRESEEA con las de otros corpus.

Los géneros discursivos, en tanto que recipientes con diversos propósitos comunicativos, con diversas convenciones de forma y contenido (Parodi, 2010) y con determinadas necesidades de imagen, pueden imponer restricciones en el uso, en la frecuencia, en los tipos y las funciones de la atenuación, que, en muchos casos, a su vez, pueden configurar patrones sociogeolectales. Algunos trabajos han intentado apuntar que la incidencia de la atenuación se asocia a las particularidades de cada género o tipo de actividad comunicativa, de forma que podamos ir poco a poco prediciendo una tipología de comportamientos atenuantes de acuerdo con los géneros discursivos. Aunque todavía son investigaciones incipientes, intentamos dar cuenta de ellas y del camino que van abriendo. Para ello, se recogen aquí los resultados de estudios que aúnan la variación diafásica con la variación diastrática y diatópica de la atenuación, siempre y cuando se comparen con los corpus sociolingüísticos recogidos en el marco de PRESEEA. Se revisan pues los estudios comparativos de los resultados del uso y funcionamiento de la atenuación o de algunos de sus recursos en entrevistas PRESEEA en comparación con conversaciones del corpus Ameresco o de otros corpus informales, y en interacciones algo más formales, como las del corpus de la norma culta (Samper, Hernández \& Troya, 1998).

Un trabajo preliminar sobre la atenuación en dos tipos de actividades interactivas y en dos dialectos es el de Albelda y Álvarez (2010) en el que se estudia este fenómeno pragmático en conversaciones coloquiales de Valencia (España) y en entrevistas sociolingüística de PRESEEA-Mérida (Venezuela). Los resultados del trabajo muestran una llamativa desproporción en la frecuencia de atenuantes en las conversaciones de España (75\% del total) frente a las entrevistas de Mérida (25\%), lo que podría apuntar tanto a variación diastrática como diatópica. No se valoran en este trabajo, sin embargo, aun recogiéndolas, las diferencias sociolingüísticas, así que hemos de esperar un poco más para tener datos relevantes al respecto. 
Entre los primeros trabajos que atienden a variación diafásica destaca el de González Riffo (2017), en el que se realiza una comparación en el uso y los valores de la atenuación en las narraciones de entrevistas sociolingüísticas del corpus PRESEEA de Santiago de Chile y en narraciones conversacionales de hablantes de la misma zona geográfica. En total, se trabaja con 90 interacciones, 54 entrevistas y 36 conversaciones, y en ambos géneros se seleccionan informantes con las mismas características sociales: mujeres y hombres, grupo de edad de 35 a 54 años y de los tres niveles de instrucción de PRESEEA. Sus resultados muestran que la atenuación se emplea más en las narraciones de las entrevistas sociolingüísticas que en las conversaciones, lo que el autor explica por la mayor cercanía entre los conversadores y la informalidad del tipo de interacción. En relación con las variables sociolingüísticas, es llamativo que en las interacciones de ambos géneros se obtengan resultados similares. En los dos corpus, las mujeres atenúan más que los hombres y los hablantes de mayor nivel de instrucción atenúan más que los de los otros dos grupos de instrucción, lo que difiere completamente del patrón sociogeolectal del uso general de la atenuación en la capital chilena y, por tanto, permite asociar el patrón divergente al tipo de secuencia discursiva: la narración. También se estudia la frecuencia de atenuación en relación con el sexo de los interlocutores que interactúan en las conversaciones; en este caso, los resultados del trabajo apuntan que se atenúa más en narraciones co-construidas por dos mujeres $y$, a continuación, en aquellas coconstruidas por mujeres y hombres y que en las que menos se atenúa es en las coconstruidas por dos hombres, lo que vuelve a acercar los resultados a patrones específicos de la narración.

Otro estudio de la atenuación en el que se compara lo que acontece en distintos tipos de actividades interactivas es el de Estellés y Cabedo (2017) sobre las diferencias en la frecuencia y distribución de los mecanismos de atenuación exclusivamente fónicos y léxico-fónicos (la prosodia atenuante se suma a formas léxicas que per se ya expresan un valor atenuador). El estudio se lleva a cabo en las entrevistas semiformales del corpus PRESEEA-Valencia y las conversaciones coloquiales de Val.Es.Co., dos géneros con diferentes grados de inmediatez. Se estudian, en total, 80 minutos de habla, en secuencias mayoritariamente narrativas. Se obtienen 338 ocurrencias de actos atenuados, realizados por hablantes de los dos sexos de los tres niveles socioculturales y de los mismos rangos que edad del PRESEEA. Los hallazgos que se destacan no se centran en la variación sociolingüística sino en la genérica, pues se concluye que las conversaciones coloquiales tienen mucha mayor presencia de atenuación exclusivamente fónica, mientras que las entrevistas semiformales presentan un mayor uso de la atenuación léxico-fónica. El trabajo demuestra que existe una relación significativa entre el tipo de atenuación y el tipo de género que contrasta con lo obtenido, en general, en el estudio de Santiago de Chile mencionado con anterioridad, lo que podría apuntar, además, patrones geolectales. 
También para el español de México, en concreto, para Monterrey, se ha estudiado la diferencia en el uso de atenuación en conversación coloquial (Corpus Ameresco, 67 conversaciones) y en entrevista (corpus PRESEEA, 108 entrevistas). Flores Treviño (2017) se concentra en el análisis de la atenuación lograda mediante marcadores de foco de exclusión ('solo', 'solamente', 'nada más', 'nada más que’ y 'nomás'). El estudio comparativo muestra diferencias en diversos aspectos lingüísticos y pragmáticos. Por ejemplo, en entrevista, la función principal de la atenuación es la autoprotección de la imagen del hablante, mientras que, en conversación, es la prevención y cuidado de la imagen del interlocutor. Asimismo, hay diferencias en cuanto al empleo de algunos mecanismos lingüísticos atenuantes: en las entrevistas son muy frecuentes las impersonalizaciones (a diferencia de lo que acontece en las conversaciones) y en las conversaciones es muy destacado el empleo de las justificaciones con valor atenuante (a diferencia de lo que ocurre en conversación). Por otro lado, al igual que ocurre en el estudio de Estellés y Cabedo (2017), los recursos atenuantes fónicos son mucho más frecuentes en conversación que en entrevista. Por último, la investigadora mexicana, aun recogiendo la estratificación sociolingüística entre los parámetros del trabajo, no ofrece resultados sociolingüísticos, por lo que debemos esperar a trabajos futuros para conocer patrones sociopragmáticos que apunten una posible variación significativa en el comportamiento de los sujetos en conversación informal y en otros registros.

El trabajo de Samper (2018), por su parte, estudia la evolución de la atenuación en una misma comunidad de habla, Las Palmas de Gran Canaria, a partir de la comparación de dos tipos de materiales muy similares -entrevistas semidirigidas- y recogidos con control sociolingüístico. Por un lado, analiza la atenuación en el Macrocorpus de la norma lingüística culta de las principales ciudades del mundo hispánico (NC), grabado en 1990 y, por otro, en las entrevistas del PRESEEA, grabadas en 2008, casi veinte años después. El estudio se lleva a cabo sobre el habla de 6 informantes de cada corpus, con iguales características sociolingüísticas. Sus resultados destacan que la frecuencia y forma de atenuación ha aumentado con el paso del tiempo: en PRESEEA se recogen un $10 \%$ de estrategias atenuantes más que en la NC. Además, se aprecia una llamativa diferencia que puede relacionarse con el factor edad: en las muestras de norma culta atenuaba más la generación intermedia, los adultos, seguida de los mayores; en el habla de PRESEEA ocurre lo contrario, atenúan más los jóvenes y la proporción va disminuyendo a medida que se aumenta en edad. También se observa la misma inversión de patrones en el caso del factor sexo: en el corpus de entrevistas más antiguo y algo más formal, la NC, son las mujeres las que más atenúan (un 11\% más que los hombres); en PRESEEA, se advierte un cambio en el comportamiento de los hombres, que elevan el número de actos de habla con atenuación (un $9 \%$ más que las mujeres), lo que podría apuntar un cambio sociopragmático en marcha y, a la vez, variación diafásica, si consideramos las muestras de norma culta como un registro algo más formal que las de las entrevistas semidirigidas de PRESEEA. Nos encontramos, 
sin duda, ante otra vía de investigación que se inicia y que, en este caso, además de dar cuenta de posible variación diafásica, ofrece información sobre procesos de cambios sociopragmáticos.

Los trabajos aquí revisados son solo algunos de los que enfocan la variación diafásica o genérica, concretamente, dado nuestro centro de atención, los que comparan datos obtenidos sobre muestras de corpus PRESEEA y de otros corpus también recogidos con control de características sociales y geográficas de los hablantes. No podemos dejar de mencionar, no obstante, que, en la actualidad, son muchas las investigaciones sobre atenuación en un tipo de actividad interactiva oral únicamente o en diferentes géneros discursivos, especialmente sobre conversaciones coloquiales, pero también sobre programas televisivos, juicios orales, debates parlamentarios, etc. Sin duda, pronto podremos tener en cuenta para la conformación de patrones de uso y funcionamiento de la atenuación en español los resultados obtenidos en estos trabajos y, dado que, en muchos casos, se controla de manera determinante la variedad geolectal de los hablantes, será factible seguir trabajando en la configuración de un mapa dinámico dialectal. Confiamos en que este futuro no sea muy lejano.

\section{CONCLUSIONES}

La serie de dos artículos (parte I y parte II) que ahora concluimos ha tratado de ofrecer el estado de la cuestión actual del estudio de la variación sociolingüística y dialectal de la atenuación pragmática en el español oral. La mayor parte de los trabajos revisados se centra en la variación diastrática y diatópica, que se han llevado a cabo en el marco del Proyecto para el estudio sociolingüistico del español de España y América, eje vertebrador. Ahora bien, también se ha dado cuenta, en el último apartado, de investigaciones que añaden la variación diafásica a los dos tipos anteriores e, incluso, la diacrónica, recogiendo las publicadas en las que se comparan resultados obtenidos en análisis de muestras de PRESEEA con las de otros corpus también sociolingüísticos.

De manera resumida, podemos decir que, al terminar la segunda década del siglo XXI, disponemos del estudio sociolingüístico completo de la atenuación en tres zonas geográficas españolas: Madrid, Valencia y Las Palmas de Gran Canaria, así como de datos parciales de Granada. Además, contamos con el estudio sociolingüístico de la atenuación en dos zonas hispanoamericanas: Santiago de Chile y Puebla de Zaragoza, en este último caso, nos falta conocer lo que acontece en el habla de los hablantes de nivel de instrucción medio. Otras áreas hispanoamericanas van avanzando en estudios del uso y funcionamiento general de la atenuación, pero aún no aportan datos completos de índole sociolingüística: conocemos lo que ocurre en el habla de una muestra de mujeres de Monterrey y de una muestra de secuencias narrativas y argumentativas en Barranquilla. 
Además, se han realizado un buen número de trabajos más particulares, microestudios, centrados en el análisis de mecanismos lingüísticos atenuantes concretos y su variabilidad sociolectal. En el habla de Madrid, se ha estudiado el diminutivo, el paralenguaje y los marcadores fático-apelativos. En el habla de Valencia, se han realizado estudios de la prosodia, de marcadores fático-apelativos y de marcadores evidenciales. En el habla de Sevilla, se han investigado también los valores atenuantes de los marcadores fático-apelativos y del diminutivo, así como el discurso referido y los pronombres impersonales. Estos últimos también se han trabajado en el habla de Granada, además de los marcadores de foco de exclusión.

Al otro lado del Atlántico, el número de investigaciones microanalíticas es bastante mayor, así como el de recursos y el de tipos de secuencias o actos de habla estudiados. En el habla de Monterrey, se ha trabajado con la atenuación y la ironía, las metáforas, los marcadores de foco de exclusión, los actos asertivos o las secuencias de justificaciones argumentativas, entre otros. En el habla de La Habana, se ha estudiado la atenuación en secuencias y actos disentivos. Por su parte, en el habla de Caracas, se han tratado los diminutivos y modificadores cuantificadores. En el habla de la Mérida venezolana, se ha analizado la prosodia atenuante. En el habla de Barranquilla, se han investigado los usos atenuantes en secuencias argumentativas y narrativas. Finalmente, en el habla de Santiago de Chile, se ha atendido la atenuación en secuencias de narración.

Los estudios macro y microanalíticos han permitido perfilar ciertos patrones sociolingüísticos y geolectales que inician la descripción de la configuración sociodialectal del fenómeno. Hemos dado cuenta de ellos en estos dos artículos, especialmente en el previo (parte I), y podemos sintetizar ahora comportamientos más generales. En Madrid, se atenúa de forma mayoritaria mediante paralenguaje, marcadores correctores y modificadores morfológicos externos. Ello contrasta con los resultados de Valencia, en donde prima la atenuación mediante marcadores de franqueza y objetividad, justificaciones y fórmulas de control de contacto. En Las Palmas, el tipo de atenuantes más frecuente es parecido al de la capital valenciana, pues mayoritariamente se atenúa mediante modificadores externos, justificaciones y fórmulas fáticas. También en Santiago de Chile el recurso más frecuente son las justificaciones, pero, en este caso, junto con los marcadores correctores y las aserciones expresadas en forma de duda o probabilidad, como ocurre en Puebla, aunque aquí son la impersonalización y la modificación morfológica los recursos que siguen en frecuencia de uso a los marcadores correctores.

El empleo más frecuente de ciertos recursos de atenuación lleva a establecer diferencias básicas en relación con el propósito con el que se atenúa en las comunidades estudiadas, lo que permite configurar los patrones referidos. Así, en Madrid, se atenúa, sobre todo, corrigiendo y reparando, y, después, minimizando y difuminando la cantidad o cualidad de lo dicho. En Valencia, se atenúa 
desfocalizando, implicando al tú y justificando. En Las Palmas, más cercana a la capital española ahora, se suele atenuar minimizando y difuminando la cantidad o cualidad, y, seguidamente, justificando e implicando al interlocutor. En Santiago de Chile, lo más frecuente es justificar y corregir con marcadores de reducción de la aserción, de forma similar y diferente a la vez a lo que acontece en Puebla, donde se corrige y se desfocaliza, con un balanceo característico entre los dos polos del continuum de máxima a mínima exposición de la imagen al atenuar.

En relación con la frecuencia de uso de mecanismos atenuantes en interacción, los estudios revisados muestran diferencias importantes en cada comunidad: en Puebla es donde se registra la mayor proporción de atenuación, con una diferencia muy grande con las otras zonas: Puebla es seguida por Valencia, Santiago de Chile, Madrid y Las Palmas.

Por último, los resultados de los trabajos revisados han mostrado que los rasgos sociales de los hablantes inciden en la conformación de subestilos comunicativos interaccionales que reflejan identidad social y dialectal. En Madrid, las mujeres, los jóvenes y los hablantes de nivel de instrucción medio son quienes mayor proyección hacia el otro manifiestan mediante el uso de la atenuación preventiva. En Valencia, este estilo interaccional enfocado hacia el otro destaca en los hablantes de nivel medio de instrucción, en adultos y mayores. En Las Palmas, este estilo discursivo es relevante en hablantes de instrucción baja y media, así como en jóvenes y adultos. En Santiago de Chile y en Puebla, por su parte, atenúan más los hombres que las mujeres y, a diferencia de lo que ocurre en las comunidades españolas, la frecuencia de atenuación disminuye a medida que se asciende en edad. Por su parte, en Santiago de Chile, los hablantes que menos atenúan son los de nivel de instrucción bajo, y en Puebla, los de nivel alto.

En el inicio de 2020, diez años después del comienzo del estudio sociolingüístico de la atenuación, tenemos un conocimiento amplio y profundo del funcionamiento del fenómeno pragmático en distintas comunidades hispanohablantes de Europa y América. Ha sido posible gracias al establecimiento de una metodología común y de su aplicación sistemática y de manera coordinada. Los resultados obtenidos en las investigaciones llevadas a cabo, a través de comparaciones, permiten comenzar a describir patrones sociopragmáticos de la atenuación y a configurar su proyección espacial, que, esperamos, dibujará el mapa dinámico que dará cuenta de la variación diatópica, diastrática y diafásica del fenómeno, y de procesos de cambio. Confiamos en que nuestro trabajo llame la atención y despierte el interés de más investigadores y sigamos avanzando en el conocimiento de tan recurrente y recurrida estrategia comunicativa. 


\section{REFERENCIAS BIBLIOGRÁFICAS}

Albelda, M. \& Álvarez, A. (2010). Los corpus discursivos en el estudio pragmático de la atenuación y de la intensificación. RILI, 16, 79-100.

Albelda, M. \& Cestero, A. M. (2011). De nuevo, sobre los procedimientos de atenuación lingüística. Español Actual, 96, 121-155.

Albelda, M. \& Mihatsch, W. (Eds.) (2017). Atenuación e intensificación en géneros discursivos. Madrid: Iberoamericana/Vervuert.

Albelda, M. \& Estellés, M. (Coords.) Corpus Ameresco. Universitat de València [en línea]. Disponible en: www.corpusameresco.com.

Albelda, M., Briz, A., Cestero, A. M., Kotwica, D. \& Villalba, C. (2014). Ficha metodológica para el análisis pragmático de la atenuación en corpus discursivos del español. (ES.POR.ATENUACIÓN). Oralia, 17, 7-62.

Álvarez, A., Blondet, M. A. \& Rojas, D. (2011). (Des)cortesía y prosodia: Una relación necesaria. Oralia, 14, 437-450.

Blanco, Y. (2014). Desacuerdo y actos disentivos en una muestra de PRESEEA-La Habana. Trabajo de Diplomado, Universidad de La Habana, La Habana, Cuba.

Briz, A. \& Grupo Val.Es.Co. (2002). Corpus de conversaciones coloquiales. Madrid:Arco Libros.

Cestero, A. M. (2014). Comunicación no verbal y comunicación eficaz. ELUA, 28, 125-150 [en línea]. Disponible en: http://rua.ua.es/dspace/bitstream/10045/48503/1/ELUA 28 05.pdf

Cestero, A. M. (2019). Apéndices interrogativos de control de contacto: Estudio sociolingüístico. Cuadernos de Lingüistica de El Colegio de México, 6(1), 1-65.

Cestero, A. M. \& Albelda, M. (2012). La atenuación lingüística como fenómeno variable. Oralia, 15, 77-124.

Cestero, A. M. \& Albelda, M. (2020). Estudio de variación en el uso de atenuación I: Hacia una descripción de patrones dialectales y sociolectales de la atenuación en español. Revista Signos. Estudios de Lingüistica, 53(104), 935-961.

Cestero, A. M. \& Rodríguez, L. (2014). Análisis de la atenuación. Guía de estudio de la atenuación. Versión julio-2008 [en línea]. Disponible en: http://preseea.linguas.net 
Estellés, M. \& Albelda, M. (2017). Evidencialidad, atenuación y descortesía en al parecer y por lo visto. Influencias del género discursivo. En B. Cornillie \& D. Izquierdo (Eds.), Gramática, semántica y pragmática de la evidencialidad (pp. 169-202). Pamplona: Ediciones Universidad de Navarra.

Estellés, M. \& Cabedo, A. (2017). La atenuación fónica en entrevistas (PRESEEA) y en conversaciones (corpus Val.Es.Co.): Un estudio de campo. LinRed, 15, 1 16.

Flores, E. \& G. Ramírez (2015). La atenuación de los actos asertivos: Diferencias entre hombres y mujeres. SOPRAG, 3(1), 90-119.

Flores Treviño, M. E. (2013). Ironización y atenuación en El Habla de Monterrey PRESEEA. En M. L. Martínez \& J. Castillo (Eds.), Memorias del VI Coloquio de Humanidades Diálogos sobre Educación, Arte, Cultura y Sociedad. Monterrey: Universidad Autónoma de Nuevo León.

Flores Treviño, M. E. (2016a). Atenuación e intensificación en el macro acto de ironizar en entrevistas orales. Letrônica | Porto Alegre, 9(1), 37-48.

Flores Treviño, M. E. (2016b). Co-presencia de la atenuación e intensificación en el uso de bastante y su derivación en el habla del noreste de México. Revista Internacional de Lingüistica Iberoamericana, XIV, 1(27), 137-156.

Flores Treviño, M. E. (2017). La atenuación y los marcadores de foco de exclusión en los corpus: PRESEEA-MTY / AMERESCO-Méx.Mty. Normas, 7(1), 19-32.

González Riffo, J. A. (2017). Estrategias de atenuación en narraciones de experiencia personal de hablantes de Santiago de Chile: Un estudio sociopragmático. Tesis de Magíster, Universidad de Chile, Santiago, Chile.

González Salinas, A. (2015). El empleo de no sé como indicador de atenuación en el grupo 1-3 del corpus MONTERREY-PRESEEA. En el $X$ Congreso Internacional da ALED. Puebla: Benemérita Universidade Autônoma de Puebla.

Guerrero, S. (En prensa). La atenuación lingüistica en el corpus PRESEEA de Santiago de Chile.

Guerrero, S., Gajardo, C., González, J. \& Reyes, A. (En prensa). Lo que pasa es que la política se ha puesto farandulera: Justificaciones atenuantes de aserciones de opinión en el corpus PRESEEA de Santiago de Chile. 
León-Castro, M. (2019). Uno como mecanismo de impersonalización en PRESEEA: Un estudio comparativo entre Granada y Sevilla. Ponenecia presentada en el II Congreso Internacional Investigando las Hablas Andaluzas, Universidad de Granada, 14 y 15 de noviembre de 2019, Granada, España.

León-Castro, M. (En prensa a). Variación en el empleo del diminutivo en el habla urbana de Sevilla: estudio sociolingüístico sobre el corpus PRESEEA.

León-Castro, M. (En prensa b). Estudio comparativo sociolectal sobre el empleo del diminutivo en la ciudad de Sevilla.

León-Castro, M. \& Repede, D. (2018). El pronombre indefinido uno como estrategia de impersonalización: un estudio en el corpus oral PRESEEA-SEVILLA. Nueva Revista del pacifico, 69, 67-89.

Malaver, I. (2018). Funciones del diminutivo en el español venezolano. Cuadernos de Lingüistica de El Colegio de México, 5(2), 5-44.

Malaver, I. \& F. Paredes (En prensa). Convergences and divergences in the use of the diminutive in Medellin, Caracas and Madrid. Spanish in Context, 17(2).

Marcano, Z. (2014). Uso atenuante de los cuantificadores más o menos, medio, un poco y un poquito en el español hablado en Caracas. Boletin de Lingüistica, XXVI (4142), 117-134.

Mondaca, L. (2017). La atenuación por medio del marcador discursivo conversacional como en el español de Chile. Tesis de Magíster, Universidad Pompeu Farbra, Barcelona, España.

Montoro del Arco, E. T. (2011). Condicionamientos sociolingüísticos de la variación fraseoléxica los operadores de foco de exclusión. LEA, 33(2), 223-264.

Montoro del Arco, E. T. (2012). ¿Son los hablantes andaluces más expresivos? La variación pragmática en el uso de los operadores focales de exclusión en Granada y Valencia. En J. A. Moya \& E. Waluch (Eds.), Español hablado. Estudios sobre el corpus PRESEEA-Granada (pp. 77-93). Varsovia: Universidad de Varsovia.

Palacios, N. (En prensa). Funciones de la atenuación y factores situacionales en PRESEEA-Puebla: Instrucción educativa baja y alta. En K. Fascinetto \& K. Cárdenas (Eds.), Tópicos de lingüística. México: BUAP.

Panussis, C. \& A. San Martín (2017). Como (que) y sus funciones discursivas en el habla santiaguina: análisis pragmático y sociolingüístico. Revista de Lingüistica Teórica y Aplicada, 55(2), 39-61. 
Paredes, F. (2015). Funciones subjetivadoras del diminutivo en el habla de Madrid. En A. M. Cestero, I. Molina \& F. Paredes (Eds.), Patrones sociolingüísticos de Madrid (pp. 117-153). Bern: Peter Lang.

Parodi, G. (2010). Academic and professional discourse genres in Spanish. Ámsterdam: John Benjamins.

PRESEEA (2003). Metodología del Proyecto para el Estudio Sociolingüistico del Español de España y de América (PRESEEA), Versión 2.0. 10-2003 [en línea]. Disponible en: http://preseea.linguas.net/Metodología.aspx

PRESEEA (2008). Marcas y etiquetas mínimas obligatorias. Versión 1.0. 31012008 [en línea]. Disponible en: http://preseea.linguas.net/Metodología.aspx

Repede, D. (2019a). Estudio sociolingüístico del discurso reproducido en el corpus oral PRESEEA-Sevilla. Signo y Seña, 35, 194-214.

Repede, D. (2019b). El discurso referido como atenuador lingüístico en el corpus PRESEEA-Sevilla. Comunicación presentada en el II Congreso Internacional Investigando las Hablas Andaluzas, celebrado el 14 y el 15 de noviembre de 2019 en la Universidad de Granada. Granada: Universidad de Granada.

Repede, D. \& León-Castro, M. (2019). Procedimientos de impersonalización en la ciudad de Sevilla: El caso del pronombre uno. Cuadernos de Investigación Filológica, 46, 81-106.

Repede, D. \& León-Castro, M. (En prensa). Aproximación sociolingüística de los impersonalizadores tú y se en el corpus oral PRESEEA-Sevilla. En M. LeónCastro \& D. Repede (Eds.), Patrones sociolingüísticos del español hablado en la ciudad de Sevilla. Berna: Peter Lang.

Reyes, C. (2014). Atenuación en narraciones coloquiales en El Habla de Monterey: El diminutivo y la risa como atenuadores ¿corteses? En M. Flores Treviño \& J. Bonfiglio (Eds.), La (des)cortesía en el discurso: Perspectivas interdisciplinarias (imagen, actos de habla y atenuación) (pp. 361-380). Monterrey-Estocolmo: UANL/ EDICE.

Rivero, X. (2011). Metáforas y atenuación desde la narración: Tres casos de El habla de Monterrey-PRESEEA. En Memorias del II Coloquio Regional EDICE México. Avances de la Investigación sobre el Discurso de la (Des) Cortesía en México, celebrado 24-27 agosto 2011. Monterrey: Universidad Autónoma de Nuevo León.

Rivero, X. (2014). La atenuación como cortesía en la narración: Metáforas y otras integraciones conceptuales. La (des)cortesía en el discurso: Perspectivas interdisciplinarias (pp. 381-403). Monterrey: UANL/ Programa Edice. 
Rodríguez Alfano, L. (2016). La atenuación en la justificación argumentativa. Un estudio aplicado al discurso de informantes de educación universitaria. Anuario de Letras. Lingüistica y Filología, IV(1), 160-195.

Rodríguez Alfano, L. (2018). La atenuación en justificaciones argumentativas en el corpus Monterrey-PRESEEA. Anuario de Letras, VI(1), 219-250.

Rojas, D., Blondet, M. A. \& Álvarez, A. (2014). Configuración tonal de la atenuación en el habla de Mérida. Lengua y Habla, 18, 93-106.

Samper, M. (2018). Un cambio en tiempo real: La atenuación entre hablantes universitarios de Las Palmas de Gran Canaria. RILCE: Revista de Filología Hispánica, 34(3),1259-1279.

Samper, M. (2019). Aproximación al uso del diminutivo en Las Palmas de Gran Canaria: datos sociolingüísticos a partir de muestras orales de actuación. En A. Cabedo \& A. Hidalgo (Eds.), Pragmática del español hablado. Hacia nuevos horizontes, (433-446). Valencia: Universitat de València.

Samper, J. A., Hernández, C. E. \& Troya, M. (1998). Macrocorpus de la norma lingüística culta de las principales ciudades del mundo bispánico. Las Palmas de Gran Canaria: Universidad de Las Palmas de Gran Canaria-ALFAL.

Santana, J. (2019). Apéndices interrogativos en el corpus PRESEEA-Sevilla: Sociolectos alto y bajo. Oralia: Análisis del Discurso Oral, 22(1).

Silva, M. (2011). La connotación en los diminutivos de El habla de Monterrey. Tesis de Magíster, Universidad Autónoma de Nuevo León, Nuevo León, México.

Torres, Y. \& Rodríguez, Y. (2017). La atenuación en Barranquilla: Estudio sociopragmático.Cuadernos de Lingüistica Hispánica, 30, 55-79.

Uclés, G. (2018). La atenuación de los marcadores de control de contacto en PRESEEA: Un estudio comparativo entre España y México. RILCE: Revista de Filología Hispánica, 34(3), 1313-1335.

Uribe, L. M. (2017). Estudio general de la atenuación: Tres casos de mujeres jóvenes en el habla de Monterrey-PRESEEA. Tesis de Magíster, Tecnológico de Monterrey, Monterrey, México.

\section{AGRADECIMIENTOS}

* Este artículo se inscribe dentro de las actividades científicas de los siguientes proyectos de investigación: La atenuación pragmática en su variación genérica: géneros discursivos escritos y orales en el español de España y América (FFI2016-75249-P), financiado 
por el Ministerio de Economía y Competitividad, el proyecto PRESEEA titulado AGENDA 2050. Procesos de variación y cambio espaciales y sociales (VARES-AGENDA 50; ref. PID2019-104982GB-C51), del Ministerio de Ciencia, Innovación y Universidades y el proyecto La población migrante de la comunidad de Madrid: factores lingüisticos, comunicativos, culturales y sociales del proceso de integración y recursos lingüísticos de intervención (IN.MIGRA3-CM; ref. H2019/HUM-5772), financiado por la Comunidad de Madrid y el Fondo Social Europeo.

\section{NOTA}

${ }^{1}$ En un trabajo posterior, Flores Treviño (2019) estudia más particularmente el marcador de foco 'nomás' en entrevistas del español de Monterrey, y entre otras, considera su función atenuante. 